\title{
INVESTIGATING ROMANTIC INTIMACY AS EXPERIENCED ON MOBILE DATING APPS: A CASE STUDY ON FLEETING ONLINE RELATIONSHIPS
}

\author{
By \\ Alicia Lapeña-Barry \\ BA, McGill University, 2020
}

\begin{abstract}
A MRP
Presented to Ryerson University

in partial fulfillments of the requirements for the degree of

Master of Professional Communication
\end{abstract}

Toronto, Ontario, Canada, 2021

(C) Alicia Lapeña-Barry 2021 


\section{AUTHOR'S DECLARATION FOR ELECTRONIC SUBMISSION OF AN MRP}

I hereby declare that I am the sole author of this MRP. This is a true copy of the MRP, including any required final revisions.

I authorize Ryerson University to lend this MRP to other institutions or individuals for the purpose of scholarly research.

I further authorize Ryerson University to reproduce this MRP by photocopying or by other means, in total or in part, at the request of other institutions or individuals for the purpose of scholarly research.

I understand that my MRP may be made electronically available to the public. 


\section{Abstract:}

Investigating Romantic Intimacy as Experienced on Mobile Dating Apps:

A Case Study on Fleeting Online Relationships

Master of Professional Communication, 2021

Alicia Lapeña-Barry

Ryerson University

This MRP explores the contemporary phenomenon of online dating in order to unearth the ways in which these dating experiences are being communicated and expressed online. By analyzing the communication of dissolved online romance as shared on YouTube, this MRP focuses on the concept of, and subsequent discourses surrounding fleeting intimacies in a modern, digital environment. Regarding the study, the author conducted a video content analysis of shared 'Story Time' videos on YouTube discussing the experience of being ghosted by a partner via a dating app. Through this research, the author found that the experience of fleeting romance is increasingly taking place in digital environments and expressed through oral storytelling practices. Furthermore, the particular gendered dynamics of the communication of fleeting intimacy are explored, with additional emphasis on the emotions felt and messages communicated with regard to the experience of ghosting. Using the sampled 'Story Time' videos as a case study, this MRP describes and analyzes the communication of modern fleeting romance in order to illustrate the increasing phenomenon of YouTube lifestyle vlogging as a form of connection, communication and sharing of intimate experiences in a digital environment. 


\section{Acknowledgements:}

I would first like to express my sincere gratitude to my thesis supervisor, Greg Elmer, for his continuous support and guidance of my MRP. Despite the ongoing Covid-19 pandemic and the barriers facing online Zoom meetings, his patience, encouragement, support and immense knowledge has helped guide me in immeasurable ways in this more than unconventional writing period. In addition to my supervisor, I would like to thank Nathaniel Laywine, my second reader, and the entire faculty of the MPC program. I am entirely grateful for all your support during this past year.

Lastly, I would like to thank all my family and friends who supported me throughout this process and in this challenging time, with a special thanks to Mom, Dad, Daniel, Lila, André, Tariq, Faith Gifford and Claire Kitching. I could not have gotten through this past year without your love and support. Thank you for everything you do for me. I am blessed to have you in my life. 


\section{Table of Contents}

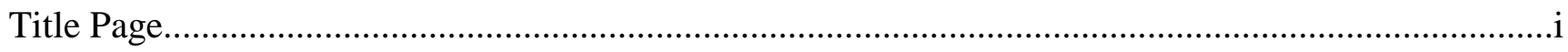

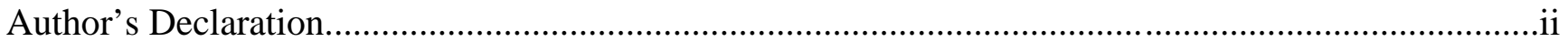

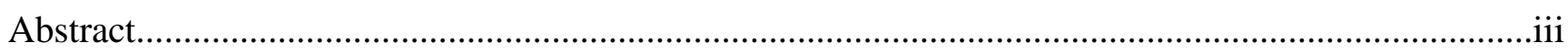

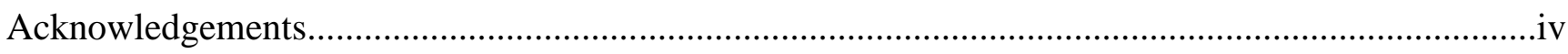

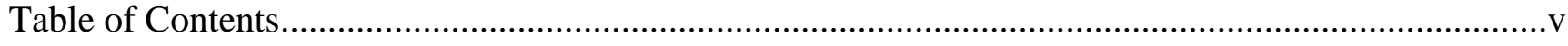

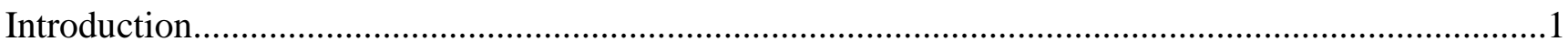

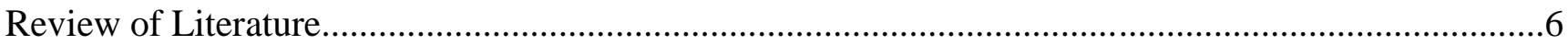

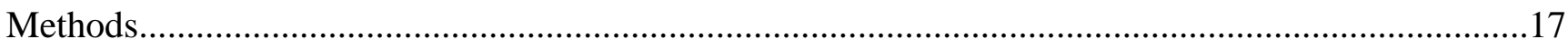

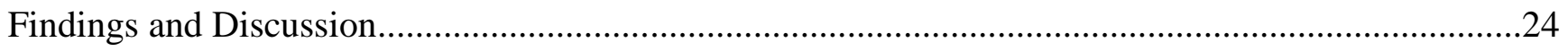

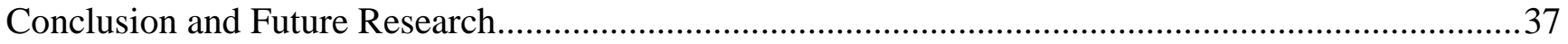

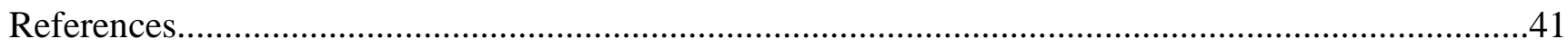




\section{Introduction}

In the past decade, the experience of romantic relationships has undergone a profound transformation. What used to be commonplace: the experience of meeting someone organically or set up by friends, has now been replaced by the experience of 'matching' with a potential suitor on a digital screen. In particular, mobile dating apps have taken over the experience of mediating intimacy, having gained increased popularity for fostering social, romantic and sexual connections between proximate strangers (Yeo \& Fung, 2018).

The popularity of mobile dating apps has been well documented. For example, a recent survey found that nearly $40 \%$ of new couples had met online (Rosenfeld et al., 2019). In addition, the Statista Market Forecast estimated that by the end of 2019, there were over 200 million active users of mobile dating apps across the globe (Statista, 2019). Further studies were conducted in different geographical and cultural contexts showing that around $40 \%$ of single adults are looking for an online partner (Gatter \& Hodkinson, 2016), and that around $25 \%$ of new couples had met through these means (Neyt et al., 2019). Based on these numbers, researchers have concluded that online dating applications play a huge and swiftly increasing role in the way people meet, effectively changing the experience of romance and dating in the modern age (Wiederhold, 2015). Furthermore, we can understand the rise in the use of mobile dating applications as altering the experience of romantic intimacy as an immediate process. As a result of the ease and accessibility with which these online interactions take place, individuals flock to mobile dating apps to form instant intimate connections. Specifically, online users seek out mobile dating apps to form quick romantic exchanges, the applications themselves noted for providing users with feelings of instant gratification, validation and connection (Castro et al., 2020).

But who, or what, is to blame for the phenomenon of online dating? The answer is more complex than it might seem. One such reason, however, lies in the popularization of the Internet. In the 
last decade, the use of the Internet and smartphones have steadily increased, giving rise to the emergence and frequent use of mobile applications (Slumkoski, 2012). The settings on these apps: often real-time, location-based settings, have resulted in more intimate and immediate formal exchanges between online users (Castro et al., 2020). A common feature on mobile dating apps in particular, these settings have significantly transformed traditional pathways of socialization and promoted new ways of meeting and/or relating to potential romantic and sexual partners (Castro et al., 2020). The ability to navigate these applications with ease, and to have immediate and unbound access to connect with other, proximate individuals, has resulted in dating scenarios that deviate from previous in-person traditions.

Furthermore, we can understand modern digital transformations as altering the experience of modern intimacy to a more instantaneous and commodified process (Kwok \& Westcott, 2020). Specifically, technological advancements such as location-based settings and ubiquitous Internet access have transformed modern courtship into a type of popular and easily accessible game (Castro et al., 2020). Where in the past, someone might rely on public settings to form and/or seek out intimate connections, they can now go online and meet someone new in the span of one second, or at the click of a button (Erevik et al., 2020). In this sense, the rise in the use of mobile technologies has produced a dating scene that is highly mediated and highly accessible, taking place largely online. Thus, as a result of technological advancements related to location-based settings in the modern age, dating apps have seemingly taken over as the primary medium to form quick and intimate romantic/sexual connections.

The popularization of the Internet and advancements in modern technologies represent one of the reasons we see a rise in the use of mobile dating applications today. Additional studies on loneliness reveal another motive for their use (Erevik et al., 2020). 
Increasingly, scholars have spoken of a loneliness epidemic across North America and prescribed this loneliness to enhanced feelings of social isolation experienced today (Apostolou et al., 2019; Sarkisian \& Gerstel, 2016). According to a study conducted by Cigna in 2018, nearly half of all Americans reported that they sometimes or always feel alone, while recent findings from Harvard University revealed that almost 36 per cent of American adults are exposed to feelings of frequent loneliness (Harvard, 2021). These findings suggest that despite the current era of increased Internetbased connectivity, that individuals continue to feel isolated and alone, reporting increasingly higher rates of loneliness and singledom across the continent (Apostolou et al., 2019).

As a result of prominent feelings of loneliness and social isolation, individuals may seek out or crave romantic interactions online (Frampton \& Fox, 2018). Given the popularity and common use of mobile technologies today, dating apps can provide users with quick and easy ways to find romantic connections and intimate partners (Erevik et al., 2020). Specifically, individuals can download a wide range of mobile dating apps and instantly connect with a number of strangers in a local context, subsequently easing their feelings of loneliness. As Erevik et al. (2020) describe, "[with online dating], individuals are no longer limited by time, social, or geographical boundaries to the same degree as offline dating" (p. 5). In addition, online dating has been noted to converge proximity, convenience, and technology to promote interpersonal interaction and facilitate physical and psychological connections (Quiroz, 2013). Accordingly, online dating may increase the number of potential romantic partners available for lonely users and be seen as an ideal medium to form intimate connections in a modern setting.

The reasons for the emergence in the use of mobile dating apps are thus well noted. In particular, the popularity of mobile dating apps can be ascribed to a more connected online world in which the use of mobile technologies is a ubiquitous and common experience. Having unbound access 
to the Internet, and the mobile applications within them, results in more active users on the dating applications themselves. Additionally, advancements in mobile-based technologies, namely: real-time, location-based settings, allow users to connect with other individuals with fewer restrictions or social barriers, making the experience of dating more accessible and easier to navigate (Erevik et al., 2020). Furthermore, the functions of ease and accessibility mobile dating apps present users with have significant ramifications for individuals seeking romantic connections. Specifically, lonely or romantically inclined individuals can use the applications to form immediate and instant connections. Rather than attempt the challenge of seeking romantic connections in person, mobile dating apps facilitate the mediation of intimacy, both on and offline.

Nevertheless, and despite current research surrounding the popular use of mobile dating apps to date, additional research surrounding the negative implications of the use of these applications specifically with regard to the experience of fleeting modern romance - is missing. Accordingly, a more thorough understanding of the phenomenon of online dating as it is communicated online - and as it responds to the experience of dissolved intimacy - is integral to the study of modern communication practices.

Given the increasing popularity of mobile dating apps to mediate romantic exchanges, this paper will explore the particular phenomenon of online dating in order to describe what happens to modern communication practices when the intimate relationships sustained on these apps cease to exist. Specifically, this paper seeks to understand how these relationships are communicated by online users once their verbal and/or written romantic exchanges become obsolete. Relying on a case study of online dating applications: Tinder, Bumble and Hinge, as well as the popular video messaging application, YouTube, an analysis of the description of fleeting online relationships within these platforms will be explored in order to describe what happens to the communication of intimacy in a 
contemporary, digital landscape. (In the context of this paper, I describe fleeting intimacy as a kind of brief romance taking place largely online and enacted through the use of a mobile dating application. Specifically, the term fleeting intimacy is used to signify acts of ghosting by an intimate online partner either before meeting up for a date, or after a maximum of two dates).

To date, there has been little consideration of the fleeting nature of intimate relationships as experienced on mobile dating apps and their direct effect on modern communication practices. However, the further study of acts of cutting-off and ghosting in digitally supported relationships, and their subsequent online and oral recounting may lead to a more thorough understanding. Considering the gaps in existing literature surrounding the use of mobile dating applications and experiences of fleeting intimacy, the goal of this paper is to investigate how ghosted partners communicate their experience of being ghosted via online, mediated networks such as YouTube. Studying these experiences may reveal heightened feelings of disconnection and loss in digitally mediated societies and lead to a more thorough understanding of the experience of modern intimacies. To this degree, an analysis of oral histories of intimacy in the form of YouTube, 'I Got Ghosted Story Times', will be explored in order to uncover the impact of fleeting online relationships as experienced on mobile dating apps.

In particular, a video content analysis of 11 YouTube lifestyle 'Story Time' vlogs will be explored, whereby the vlogger is discussing their experience of fleeting intimacy as mediated online and through a dating app. As such, the video content analysis will serve as the focus of the author's study, with additional coding exercises taking place in order to uncover what emotions are felt and what messages are being communicated by these vloggers with respect to the experience of fleeting romance. In performing a study of this nature, the author anticipates uncovering how the communication of modern romance is expressed online, as well as how experiences of fleeting online 
romance inform and shape the participation in, and experience of modern dating in a contemporary, digital environment. Understanding the way fleeting intimate relationships are conceptualized by these YouTube lifestyle vloggers once they have ended is integral to broadening the literature surrounding romantic exchanges and, furthermore, unearthing key insights into the experience of modern, digitally mediated intimacies. Therefore, the following research question is raised:

(RQ1): How do individuals communicate and/or respond to acts of ghosting in an online, digital context?

The above research question aims to answer how the use of mobile dating apps is taken up and described by lonely online users, as well as to answer which forms of affect are produced by the experience of fleeting online romance, and their subsequent influence on dating experiences in a modern setting. An analysis of this nature may reveal significant insights into the way humans relate, understand, and interact with one another in a contemporary digital environment, with specific regard to the experience of online dating.

\section{Review of Literature}

Several bodies of literature inform a study of this nature. The first has to do with the experience of online intimacy, and subsequent fleeting romantic exchanges.

\subsection{Digital Romance}

Engagement in intimate online relationships is increasingly commonplace today (Hogen et al., 2011). As a result of the pervasive use of mobile applications, it is now easier than ever to network, connect and form relationships with individuals online (Sutcliffe et al., 2018). In particular, scholars have described the technological affordances offered by mobile applications, be it: instant messaging, location-based settings or video-chatting, to foster increasingly connected and personal social 
interactions in both local and global contexts (Lomanowska \& Guitton, 2016). Furthermore, research suggests that the use of mobile applications has revolutionized the way individuals experience intimacy and connection in a modern setting; by breaking down pre-existing social barriers and allowing constant and convenient communication in a global environment (Wooley, 2013). According to Chen (2012), modern innovations in digital media have taken communication practices to a higher interconnected and complex level, by allowing communication to be constant, convenient and readily available. In regard to the experience of modern romance, the implications of constant, convenient and readily available digital communication among online users results in an increasing number of individuals mediating and sustaining relationships online, confirming the notion that the popularity of cyberspace interactions and relationships in the U.S. and other countries has steadily increased in recent years (Yum \& Hara, 2005).

Regarding the experience of modern digital intimacy, Hardey (2004) describes the Internet as a mode of meeting people and forming relationships, wherein information and communication technologies supplement or replace traditional routes to potential romantic encounters. As a result of the ability to engage with a plethora of individuals with little-to-no social barriers or interference, mobile devices have made communication practices between individuals faster and easier than ever (Ozë, 2017). In particular, the ability to connect and communicate with a wide range of individuals through an easily accessible and interconnected online medium - has been noted to foster increased personal, romantic and intimate exchanges (Sutcliffe et al., 2018). As Kwok \& Westcott (2020) further describe, the experience of participating in these personal and intimate online exchanges often results in profound feelings of connection and love.

This research confirms the contemporary notion that mobile applications have the unique ability to mediate romantic exchanges on an unprecedented level; through the dissolution of previous in- 
person, social barriers (Ozë, 2017). Accordingly, we can understand mobile applications to facilitate intimate interactions by making them more instantaneous and less socially complicated to navigate (Wooley, 2013). Furthermore, these findings suggest that the use of mobile technologies have effectively altered the experience of modern romance; through recent technological advancements in which access to other online individuals is highly accessible and highly convenient (Lomanowska \& Guitton, 2016, Chen, 2012). However, an analysis of what happens to digital romances once their intimate communications cease to exist remains missing from the current body of literature.

Consequently, it should be noted that the widespread integration of mobile technologies into everyday lives continues to alter the practical modes through which individuals engage and communicate with one another in a daily setting (Lamonowska \& Guitton, 2016). Despite the affordances of connection mobile technologies bring to its users, it remains easier than ever to disconnect and distance oneself from intimate, online connections (LeFebvre et al., 2019). Specific technological features supported by mobile-based technologies help facilitate acts of blocking and cutting off, subsequently helping to delete any semblance of an online digital footprint and/or experiences of intimate online relationships (LeFebvre, 2017). This, in turn, pointing to a new and increasingly popular phenomenon of relationship dissolution as enacted online.

\subsection{Fleeting Online Relationships}

The impact of fleeting relationships in digital contexts has seldom been explored. However, certain scholars have begun to broach the topic in relation to specific digital acts of 'cutting off'.

In particular, scholars have spoken of the social phenomenon of relationship dissolution as increasingly taking place online, and enacted through mobile technologies such as text messaging, email, and social networking sites (Delevi and Weisskirch, 2013). A common practice today, digital users will rely on technological interfaces to end their relationships or cease communications with their 
romantic partners, rather than resolve the conflict in-person (LeFebvre et al., 2019). Moreover, the reasons for opting for a digital relationship dissolution strategy may vary. However, current research suggests that the frequent use of mobile technologies to mediate and sustain intimate relationships results in individuals favouring technology-mediated dissolution strategies in today's modern digital age (Delevi and Weisskirch, 2013).

As Finkel et al. (2012) explain, the increasing reality of romantic relationships being mediated online results in fundamentally different social outcomes within the context of dissolved intimacy. In particular, the frequent use of mobile applications as a primary medium of communication and connection results in more relationship dissolution taking place online and subsequently, more fleeting romantic and intimate exchanges (Delevi and Weisskirch, 2013). Today, online users can easily block, delete, unfriend, unmatch and ghost their contacts or partners, making the experience of online intimacy an increasingly risky and fleeting game. Despite the ease with which individuals can now connect and form intimate online relationships, these very relationships can just as easily be taken away, resulting in more temporary and transient romantic interactions. The ability to disengage from intimate relationships at the click of a button and/or through a specific online medium represents a new social phenomenon in which acts of conflict resolution are increasingly traded in for more transient, temporary and avoidant digital acts.

Sociologists Calvin Morill, David Snow and Cindy White (2005) have spoken of the phenomenon of fleeting intimate relationships, describing them as brief interactions coloured by emotional dependence and intimacy. In the digital context, intimate relationships are often and equally emotionally dependent and intensive exchanges. However, specific technological 'cut off' features enable particular fleeting romantic interactions, subsequently altering the landscape of romance in a modern digital setting (Delevi and Weisskirch, 2013). To this degree, we can understand the 
dissolution of online relationships by acts of ghosting, unfriending and/or unmatching as intimacy avoidance via distant communication (Delevi and Weisskirch, 2013). In particular, the mechanisms of exchange present on online platforms have been noted to both enhance and facilitate avoidant communication practices and disconnected relationships in a modern setting. As a result of distinct 'cut off' features, the use of mobile applications to mediate intimate and romantic exchanges can thus be understood as fleeting in nature (Delevi and Weisskirch, 2013).

As such, the above research suggests that the frequent use of mobile applications as a primary mode of connection and communication is rapidly altering the modes with which individuals communicate and engage with one another online. In turn, suggesting that individuals are increasingly engaging in avoidant and disengaged relationship dissolution practices in a digital context.

Despite these findings, minimal research has focused on how the specific fleeting affordances present on mobile dating apps affects the communication of modern romantic intimacy. A more thorough analysis of acts of blocking, unmatching, and ghosting online is necessary in order to consider the direct impacts of fleeting romance on modern, digitally supported relationships. Though surrounding literature on the nature of fleeting online relationships is briefly discussed, an analysis of the specific ephemeral affordances of connection/disconnection on mobile dating platforms is needed.

\subsection{Technological Affordances of Ghosting}

Current literature supports the idea that the dissolution of relationships is increasingly taking place online and in digital contexts (Delevi and Weisskirch, 2013). Furthermore, the phenomenon of ghosting has been noted as a primary form of relationship dissolution in the digital age (LeFebvre et al., 2019). But what exactly does the act of ghosting mean, and what does it represent in the context of intimate online relationships? 
Having gained traction throughout 2014 and 2015, Everingham (2019) describes the emergence of ghosting as a relatively new phenomenon in today's contemporary society, "made easy by our use of communication technology" (p. 1). In particular, ghosting consists of "unilaterally ceasing communication (temporarily or permanently) in an effort to withdraw access to individual(s) prompting relationship dissolution (suddenly or gradually) commonly enacted via one of multiple technological mediums" (LeFebvre et al., 2019, p. 10). As Lefebvre (2017) describes, ghosting is an act of intimacy avoidance enacted via the use of technology. When someone is ghosted, the ghosted partner (ghostee) does not know what has happened within the relationship, is left to try and understand what the partner's lack of communication means, and is unable to get proper closure (Lefebvre, 2017). Rather than provide a partner with a verbal explanation for the break-up, ghosting has emerged as a popular technologically mediated relationship dissolution process that favours indirect action and/or communication (Punyanunt-Carter, 2017). To this end, the statistics supporting the commonality of ghosting in today's modern relationships are well noted.

A recent study found that out of a sample study of 13,000 participants, around a quarter of participants had been ghosted by their romantic partners, while one-fifth reported ghosting a romantic partner themselves (Freedman et al., 2018). In addition, a 2018 survey found that 65 percent of the study participants reported previously ghosting a partner, while 72 percent reported that their partner had ghosted them (Koessler et al., 2019). In another, more recent study, researchers identified ghosting as a highly prevalent relationship dissolution strategy in North America, with prevalence rates ranging between $13 \%$ and $23 \%$ for adults in the United-States (Navarro et al., 2020). The reasons for ghosting a partner may vary. However, research has found that one of the primary reason's individuals choose this relationship dissolution strategy is that ghosting someone is easy, which increases the chances of this strategy being employed without consideration of the possible downstream consequences (Freedman et al., 2018). Thus, despite the minimal conflict resolution the act of ghosting entails, and the 
psychological harm it might cause the 'ghostee', the ability to avoid direct conflict through this particular action is often favoured in today's digital age (LeFebvre et al., 2019).

The degree with which ghosting has emerged as a popular form of relationship dissolution is made clear in recent studies. Despite the concerning trends surrounding an increase in acts of intimacy avoidance, these statistics usefully reveal the extent to which ghosting plays a major role in today's breakup strategies. Furthermore, and integral to a study of this nature, is an understanding of the role technology plays in both the mediation and dissolution of modern relationships.

In the context of experienced online intimacy and reverting back to the first section of the literature review, research has confirmed that the initiation, development, and maintenance of relationships has significantly been impacted by technology (Sutcliffe et al., 2018). In a world where more and more individuals are seemingly connected and 'online', it is no surprise that the Internet and mobile-based technologies have facilitated online interactions and romantic exchanges through their easily accessible and connected settings. However, as many relationships start via the use of technology, the ability to dissolve them via the same medium becomes more and more desirable (LeFebvre et al., 2019) In particular, the common use of mobile technologies to mediate relationships have accelerated acts of intimacy avoidance and relationship dissolution through specific of acts of ghosting. Furthermore, by avoiding phone calls and texts, unfollowing, unfriending and/or blocking a partner, the mobile technologies used to support romantic connections are easily severed in a contemporary setting (Freedman et al., 2018).

Thus, as a result of an increased culture of conflict avoidance, an increasingly connected online world, and its functions of ease, acts of ghosting have become a favoured relationship dissolution strategy in the technological age (Lefebvre et al., 2019). However, the way individuals deal with and communicate the act of being ghosted in a modern/digital setting is still missing from current research. 
Increasing accounts of being ghosted necessitates further research in order to understand exactly how modern technology is affecting the way relationship dissolution occurs in a contemporary setting. Further understanding of the history of oral communication practices, as well as an analysis of YouTube Story Times as an effective medium of modern communication may help us come close.

\subsection{Histories of Oral Communication}

For years, humans have been using oral communication methods as a means of recounting personal histories and intimate exchanges (Ritchie, 1995). Such methods have usefully revealed the ways in which "particular people recall past livelihoods, conflicts... self-conceptions, and social practices" (Giles-Vernick, 2006, p. 1). In the context of producing an epistemic rhetoric, the process of sharing oral histories offers rich meaning to personal experiences, due to their subjective nature (Langellier, 1989). By drawing on the memory of these subjective experiences and communicating them in an oral fashion, individuals are able to craft deep personal narratives with significant emotional weight and personal legitimacy (Anderson, 2010). In such a way, the act of oral communication practices represents an active process of meaning making as based in individual memory (Ritchie, 1995). Through the act of remembering, individuals draw on their personal experiences to communicate both historical and epistemic truths; through the medium of the life story (McAdams, 1999). It is then through these oral histories and life stories that individuals can pass on knowledge, memory and experience (Collins \& Gallinat, 2013).

Research has confirmed oral histories as a useful tool of epistemic communication. On the level of an effective medium of communication, Thompson (2017) has described oral histories as a significant and transformative tool in all societies, in that oral traditions "open up" important new areas of inquiry into the lives of individuals on a wider and more relational scale. Specifically, Thompson highlights oral communication practices as practices which a) build community by the sharing, 
dissemination and relating of personal experiences between individuals, b) disrupts colonial narratives by broadening the number of historical voices and histories shared in the historical canon, and c) presents more opportunities for radical or subversive social and political commentary from a personal and epistemic grounds. To this degree, we can understand oral traditions and the transmission of life stories as maintaining a historical record and sustaining culture and identities (Hanson, 2009). In particular, oral traditions are noted for their functions of sharing, communicating identity, preserving history and disseminating cultural knowledge between groups and individuals.

Insofar as persevering the history and experiences of the self, indigenous theories on oral traditions serve as a useful starting point for further conceptualizing the significance of oral histories when applied to a contemporary, digital setting.

As scholars Renée Hulan and Renate Eigenbrod (2008) describe, oral traditions are the means by which knowledge is reproduced, preserved and conveyed between generations and form the foundation of Aboriginal societies by connecting speaker and listener in communal experience and uniting past and present in memory" (p. 7). According to Hulan and Eigenbrod, oral traditions represent one of the dominant systems used to convey knowledge and communicate both personal and historical experiences with others in the indigenous community. Furthermore, they represent a system of epistemic knowledge production which works to connect and unite individuals on a grander and more relational scale (Augustine, 2008). As Margaret Bruchac (2014) explains, Indigenous oral traditions "constitute a form of traditional knowledge that can teach, carry, and reinforce other knowledges" (p. 6). In addition, indigenous oral traditions "often contain insightful explanations that focus on details" with information typically passed on through "acts of storytelling.... As they verbally reconstruct connections with the past" (p. 6). Thus, on a practical level, oral traditions are effectively utilized in indigenous communities to transmit important histories, stories, knowledge and teachings to others 
(Basso, 1996). They are equally used to teach others about historical and personal experiences, as well as to form a connection to the past and to present community (Cruikshank, 2014). ${ }^{1}$

The above research suggests that oral histories serve as a significant primary source for people wanting to discover more about a certain event or era, by providing an insight into the impact events had on the people involved. Through oral communication measures, individuals consolidate and reconsolidate their experiences through acts of storytelling, in turn, giving personal significance to their intimate exchanges (Ritchie, 1995). This method of conceptualizing oral communications is thus fundamental for the project moving forward. Understanding the way oral practices of transmission are used to further conceptualize and preserve one's personal experiences, and/or produce relatable content to share with others is significant. Though little-to-no research has focused on the significance of oral histories in the context of fleeting online relationships, initial research on the significance of oral histories provides a useful framework for studying the proposed research question. Furthermore, a more thorough analysis of the nature and power of oral histories on YouTube may reveal significant insights into how intimate online exchanges are communicated once they are no longer present 'on the screen'. Moving forward, the remainder of the paper will be dedicated to unearthing exactly how the process of oral storytelling is used online to conceptualize fleeting intimacies in a modern setting.

\footnotetext{
${ }^{1}$ Although the practice of orality does not represent a comprehensive method of understanding indigenous culture, history and practices (a history/culture that is so rich and experienced through embodied and historical truths/experiences), the particular focus on knowledge transmission through oral methods/the oral sharing of personal experiences is useful in the context of the project moving forward.
} 


\subsection{YouTube 'Story Times' as a Medium of Communication}

To date, there is no direct literature surrounding the implications of YouTube 'Story Times' as an effective medium of contemporary communication. However, recent studies surrounding the act of vlogging and digital storytelling on YouTube serves as a useful point of departure in relation to the proposed research question.

To this degree, scholars have confirmed acts of digital storytelling as an emerging and increasingly popular mode of communication in the digital age (Dreon et al., 2011). Described as an act of combining narrative with digital media such as images, sound, and video to create a short story (Robin, 2008), digital storytelling is one of the most common modes of interpersonal communication present on the video application to date (Dreon et al., 2011). Noted as one of the most popular forms of digital storytelling, 'lifestyle vlogging', in particular, has been studied as a popular means of expression and connection in today's digitally mediated societies (Ashton \& Patel, 2018). By uploading digital stories and videos to their YouTube accounts, vloggers can produce personal narratives for their followers through the medium of the vlog, as well as interact with followers through comments, ratings, or video responses (Robin, 2008). In such a way, digital storytelling in the form of lifestyle vlogging has become a primary mode of building connection and community within today's digital landscape and globalized society, at large (Lang et al., 2019).

In addition to uploading general lifestyle videos, vloggers often share and participate in particular video genres and trends. One such video genre is the 'Story Time' video. As journalist Emily Brown (2017) explains, "Story Time videos are winding monologues about a salacious event in someone's life" (p. 1). Averaging at a length of between 15-30 minutes, 'Story Time' videos have increasingly been noted for their intimate form of storytelling, with vloggers divulging personal and intimate details about their experiences and lives. This kind of divulging of personal details has been 
described as a form of affective communication between vlogger and audience, in which the YouTuber forms and produces emotional exchanges through the sharing of personal and often 'juicy' information (Berryman, 2020). In such a way, YouTube lifestyle vlogging and the various video trends users participate in has become a primary communication channel for digital users. In particular, the 'Story Time' genre allows individuals to put themselves on camera and talk, to self-express, and participate in community activities through the medium of the vlog itself (Bernard, 2020). In the digital context, YouTube lifestyle vlogging has thus come to represent an increasingly popular means of expression, connection and community in the modern digital age (Berryman, 2020).

Conceptualizing the act of vlogging on YouTube as a medium of connection and expression, specifically through the 'Story Time' genre, is necessary for the project moving forward. Despite evident gaps in the above literature, these conceptual topics represent an initial framework for conceptualizing and, further, theorizing a project of this nature. Moving forward, the author aims to bridge any gaps between the missing literature surrounding fleeting romances and their subsequent online recounting. Specifically, the author aims to answer how individuals deal with and communicate the act of being ghosted when using a digital platform as their primary medium of connection and communication.

\section{Methods}

- Aims

To begin the study, the author posed the primary research question: (RQ1) "How do individuals communicate and/or respond to acts of ghosting in an online, digital context?". By posing a question of this nature, the author aimed to investigate exactly how contemporary discourses were formed, and on which online mediums they were circulated on with regard to the experience of dissolved romantic relationships. Accordingly, the primary aim of this study was to examine how fleeting relationships 
were conceptualized and communicated in a modern, digital setting. More specifically, the author aimed to target the online storytelling practices of ghostees making narrative sense of their ghosting experiences as it related to the experience of intimacy as mediated on a mobile dating app.

Using this research question as a point of departure for the study, several philosophical considerations arose for the author throughout the project. The author began to question whether a broad analytical approach would be able to accurately respond to the proposed research question. With the primary aim of the study being to investigate the modern, digital communication of fleeting online romance, certain spatial and gendered examinations thus came to light which further informed the project. Such spatial and gendered considerations resulted in the following/additional research questions:

RQ 2: "Where is the communication of fleeting online relationships taking place?"

Sub question: "How are fleeting relationships being conceptualized in these spaces?"

RQ 3: "Is there a gendered discrepancy between who is ending online relationships, causing them to become fleeting in nature?"

Sub question: "What can a gendered analysis of fleeting relationships tell us about the experience of modern digital communications?".

With these three research questions in the mind, the aim of the project remained the same, albeit with a more nuanced, gendered and spatialized/local lens. The goal of the project was to investigate how, where, and by whom the experience of being ghosted by a romantic partner was communicated.

\section{- Research methods}

The author used quantitative research methods to conduct the study. Specifically, a video content analysis was selected as the primary research method. Going further, an analysis of YouTube 
'Story Time' videos by individuals discussing meeting their partners on mobile dating applications (Tinder, Bumble \& Hinge), and subsequently ghosted by said partners was surveyed. The methodological strategies employed in the video analysis are defined by the implementation of certain search terms, a specific timeline search and the use of a coding sheet, all of which are discussed in further detail below.

\section{- Search Terms}

When beginning to conceptualize the initial methodological framework for the study, the author had intended to do a large literature review of published academic works discussing the use of mobile dating apps and fleeting relationships. To more thoroughly get a grasp on what relevant written material was out there, the author used various academic search engines such as Google Scholar and the Ryerson University Library website. The intent in using these search engines was to find existing literature surrounding the phenomenon of ghosting as experienced on mobile dating apps, and to find subsequent studies revealing the impact of technology on contemporary relationship dissolution strategies. In terms of finding relevant literature, the author came up with some preliminary search terms to input into the academic search engines. The search terms were as follows: "fleeting relationships", "fleeting online relationships", "fleeting technologies", "ephemeral online relationships", "ephemeral online communication", “online ghosting”, "ghosting online dating", "dating apps and relationships". The author selected these search terms as they felt the terms would lead to an expansive overview of literature surrounding the phenomenon of online relationship dissolution strategies, as well as provide well-rounded theoretical explanations to how individuals communicate the act of ghosting and/or being ghosted.

However, the existing literature related to these search terms was scarce and did not lead to any immediate conclusions or answers surrounding the initial research question: "How do individuals 
communicate and/or respond to acts of ghosting in an online, digital context?". The lack of direct literature and/or studies related to ghosting and mobile dating apps pointed to a prominent gap in the communication of modern fleeting intimacies. Accordingly, the author's research interests began to change.

As a result of the lack of written academic material relating to $\mathbf{R Q 1}$, the author began to investigate other contemporary mediums of communication for analysis. Upon abandoning the idea of an extensive literature review for the project, the author was reminded of YouTube 'Story Time' vlogs as a popular digital medium used to communicate personal experiences related to modern dating. Accordingly, the author's research interests shifted toward a video-content analysis of the YouTube 'Story Time' genre. The author chose this method as they felt it would more accurately respond to the initial research question and provide first-hand, epistemic narratives surrounding the experience of modern, fleeting intimacies. To begin the video-based research, the author had to refine their search terms from previous attempts in the academic search engines, to more straightforward search terms on YouTube. Accordingly, the author came up with a new set of predetermined search terms to input into the YouTube search bar. The search terms were as follows: "I Got Ghosted Story Time”, "I Got Ghosted on Tinder", "I Got Ghosted on Hinge”, "I Got Ghosted on Bumble”, and "Ghosted Dating Apps".

These specific search terms were then applied to a YouTube video search and allowed the author to more easily sort through video content and refine their overall research. The search terms were chosen as the author felt that a more direct and less broad verbiage would present them with more relevant and insightful video content in relation to the above research questions. Moreover, all videos applicable to these specific search terms and with relevant titles were viewed and considered for the study. Out of thousands of videos uploaded to the platform, 11 'Story Time' videos were selected as 
relevant to the study and capable of responding to all of the research questions. Regarding a more extensive rationale for their selection, the videos were chosen as a result of their direct relevancy to being ghosted by romantic partners met on particular dating apps (Tinder, Bumble, Hinge), as well as their direct online communication of the act of being ghosted on a digital platform (YouTube). In total, 8 vloggers discussed their fleeting relationships while using Tinder, 2 vloggers discussed their fleeting relationships while using Bumble, and 1 vlogger discussed their fleeting relationship using Hinge.

\section{- Timeline}

A timeline of videos ranging from the years 2016-2021 was selected. The rationale for this timeline became more apparent to the author throughout the study. Specifically, the years 2018-2020 were highlighted as particularly popular years to upload 'I Got Ghosted' YouTube 'Story Time' videos, with the years 2016 and 2021 offering minimal, albeit relevant and insightful content for analysis. As such, any video preceding the year 2016 was disregarded, as the author did not want to stray too far away from either current or more relevant videos of vloggers discussing their ghosting experiences. In addition, the author did not want to include data from vloggers that were no longer active on the platform. Including videos by active vloggers was integral to the study in order to keep the video narratives relevant to the discussion of ghosting and contemporary digital communications.

\section{- Coding Sheet}

With both search terms and relevant timelines applied, and 11 YouTube 'Story Time' videos selected, the author was able to move forward to the coding portion of the study. Specifically, once a video was determined to be of relevant insights for the study, the author sorted through the selected number of videos and further analyzed them through a video content analysis and coding sheet, which provided well-rounded explanations to the proposed research questions. 
The coding sheet was designed to follow a specific search criterion set up by the author. Such search criteria included the following coding sheet categories: 1) the URL of the video, 2) the title of the video, 3) the length of the video, 4) the date posted, 5) the username of the vlogger 6) the number of comments on the video, 7) the gender of the vlogger, 8) the location filmed, 9) the messages communicated, and 10) the emotions felt. These categories were determined and input into the excel sheet for reference purposes for the author, as well as to make note of any potentially relevant information with respect to the proposed research questions. Regarding the URL of the video as well as the username of the vloggers (categories 1 and 5), this information was coded for the author to go back and review the content and messages presented by the lifestyle vlogger and furthermore, to ensure they were an active participant on the YouTube app. Keeping track of this information provided a valuable tool for the author when constructing their findings, as well as for additional reference purposes. In addition, the active participation of vlogger on YouTube was a seminal search qualification for the author when constructing their research. The author wanted to ensure the selected videos were coming from active individuals discussing these topics with at least a minimal YouTube audience. The emphasis on this selection was to keep the findings contemporary, relevant and informed.

Coding the title and length of the videos (category 2 and 3) allowed the author to make more thorough links between the messages being communicated and shared online in relation to the research topic and specifically, under which terms, and for how long, individuals were communicating the act of being ghosted. Similarly, coding the amount of comments (category 6) allowed the author to further understand the relational process of sharing 'Story Time' videos and the ways in which discourses surrounding being ghosted/fleeting intimacies were being formed online and on the YouTube platform, specifically. This would help the author to respond partly to RQ1. Perhaps the most significant point of analysis, categories 7-10 would provide the most vital data points for the author to consider. Category 7 was intended to respond directly to $\mathbf{R Q 3}$ and its sub question, while category 8 was intended to respond 
directly to RQ2. In addition, categories 9-10 were designed to answer the emotional and conceptual sides of the study, as an attempt to understand how YouTube vloggers were conceptualizing acts of fleeting or ephemeral romances, and what this might reveal in terms of the communication of romance in a contemporary and digital setting.

Ultimately, these categories were investigated in order to consider exactly how, who and where the act of being ghosted was being communicated online. The primary goal of this research was to fill out each category in order to form an informed response to the proposed research questions. By looking at various social, cultural, location-based and gender-based markers, the author was able to more faithfully respond to RQ1-RQ3. Moreover, from this initial coding process, the author was able to draw some preliminary conclusions regarding the online communication of being ghosted. Such conclusions were that a) individuals were using digital video messaging platforms to communicate the act of being ghosted and b) a disproportionate amount of women vloggers were communicating their experience of being ghosted by their partners on the YouTube platform in comparison to men. This initial data based on preliminary coding evidence would be further explored and substantiated in subsequent findings.

\section{- Further explanation of research methods chosen.}

In terms of opting for a video content analysis, the rationale for the selected research method lies in the unique ability for YouTube vloggers to discuss personal and unfiltered experiences through the sharing of their videos. Specifically, the author saw the video messaging platform as an ideal medium to analyze first-person narratives with direct relevancy to the proposed research questions in a methodological and systematic manner. In addition, these videos were chosen for the commonalities present in their story time messaging, which the author decided must have either partly or directly communicated the experience and emotions surrounding being ghosted by a partner, and its subsequent 
relevancy to today's digital age. Furthermore, by viewing personal vlogs dedicated to discussing the experience of being ghosted, the author wanted to leverage and further investigate the power and practice of oral histories of transmission via a digital medium such as YouTube, as well as understand how and which online users were leveraging digital platforms to communicate the experience of fleeting romantic relationships. In particular, the author found that the rise in popularity of lifestyle vlogging in recent years would provide a useful primary source to investigate the research topic of fleeting online relationships, with certain vlogs providing key, epistemological details related to the research questions.

Additionally, the author found that the practice of coding the videos would allow them to put forward a constructive argument on the effects of fleeting relationships in a digitally mediated world. By implementing certain search criteria and coding the videos accordingly, the author could narrow in and focus on certain conceptual topics and frameworks which would lend themselves to further conceptualizing the experience of modern fleeting intimacies. Specifically, by focusing on certain video demographics related to age, gender and location of the vlogger, as well as the message characteristics and strategies of these YouTube vloggers, the coding sheet and video analysis would provide a systematic portrait of the communication of fleeting intimacies in a digital context.

Owing to limited insights in relation to the topic of fleeting intimacies, the author felt that an exploratory project of this nature would reveal significant insights into the way modern communication practices are enacted in the digital sphere.

\section{Findings}

Taking us back to RQ1, the author was interested in the question: "How do individuals communicate and/or respond to acts of ghosting in an online, digital context?". By posing a question of this nature, the author sought to identity, and further investigate, practices of digital storytelling in 
relation to the contemporary experience of fleeting intimacy and the particular use of mobile dating apps. Responding to the lack of current research surrounding the topic, the author saw this gap as an ample opportunity to study the phenomenon of ghosting in a contemporary setting, as well as investigate modern communication practices in an online environment.

Throughout the video content analysis and in researching this first question, the author came to some salient findings. To begin, the author was able to identify the YouTube platform as one of the primary digital platform's individuals are using to communicate acts of ghosting. In particular, the author found that online users were commonly employing digital video messaging applications, and specific video genres within them, to communicate the practice of ghosting/being ghosted. Whether a 'Story Time' video or an informational/self-help video, digital video mediums were found to be the most common method of digital communication with regard to the specific phenomenon of ghosting and today's modern relationship dissolution strategies. Accordingly, these initial findings reflect the notion that digital media are increasingly becoming the dominant modes through which individuals relate, interact and communicate with one another in a contemporary setting (Burgess \& Green, 2018). As scholars Paul Haridakis and Gary Henson (2009) explain, online mediums such as YouTube are increasingly favoured and made dominant in today's digital societies, for their unique ability to seamlessly move between "traditional mass communication activity of watching mediated content, and interpersonal or social connection activity of sharing it with others" (p. 318). In such a way, the common use of the YouTube platform to communicate acts of ghosting is unsurprising.

We can understand the popularity of both uploading and consuming videos on YouTube as a cause of the platform's features as a medium of mass open communication, as well as its fostering of online communities. The author's initial findings on where the digital communication of ghosting is most commonly discussed confirms this. An additional understanding of YouTube's features of open 
communication and online community further helped the author respond to the initial research question.

In responding directly to $\mathbf{R Q 1}$, the author found that individuals specifically communicate and respond to acts of being ghosted by using oral communication methods. In particular, individuals use and rely on digital mediums, such as YouTube, to orally transmit life stories and furthermore, make sense of and communicate the experience of their fleeting relationships. By employing the 'Story Time' video genre, online users make use of the practice and transmission of oral histories as a means of self-expression, recounting and conceptualization of these relationships. In such a way, these online users/lifestyle vloggers rely on traditional practices of oral communication as a means of both selfexpression and knowledge transmission through the act of digital storytelling. Specifically, YouTube vloggers build on their own experiences and communicate them in an oral fashion to convey epistemic knowledge to their subscribers and/or a larger YouTube audience. By sharing their personal experiences of ghosting through the oral practice of 'story telling', these YouTube vloggers are able to transmit knowledge to other online users watching their videos, in turn, inviting collaboration and community with their audience (Rotman \& Preece, 2010). Accordingly, these findings reflect initial literature review research in which the practice and transmission of oral histories serves to communicate both personal or historical experiences with the self (vlogger) and with others (viewers). As a result, the communication of fleeting romance becomes a sharing ritual/practice in the digital context.

Ultimately, the author's research found that individuals respond to being ghosted by creating YouTube 'Story Time' videos. Specifically, the dissemination of oral histories in the form of digital storytelling is used as a primary mode of communicating fleeting intimacies in a modern, digital context. 
What is perhaps most striking in these findings is the almost paradoxical reality of using an online medium to communicate acts of digital ghosting behaviour. Rather than relying on family members, friends, a therapist, or various in-person modes of communication to make sense of the fleeting online relationship, these vloggers have opted for a digital medium to communicate the personal experience of being ghosted from a partner on a mobile dating app. Further proving that online mediums have become the new mode of sharing and connecting in a contemporary landscape (Pittman, 2015). Moreover, given that there is an entire subsection of YouTube videos dedicated to the 'I Got Ghosted: Story Time' genre, these findings may point to a new, marked experience of sharing and community and in a contemporary context, in which the experience and communication of intimate and fleeting relationships is increasingly taking place in a mediated and digitized environment. The comments under these videos further substantiate these findings. Raging from 5-100 comments per vlog, the comments under these vlogs act as a kind of extension of an oral dialogue between both the vlogger and their audience, in which the conversation of fleeting romance is further discussed and related to within the comment section. In such a way, we can interpret the medium of the lifestyle vlog/oral communication practices on YouTube to represent a new mode of sharing and relating to others in today's digital societies.

The reasons that vloggers opt for the digital communication of their fleeting relationships remains of interest for the author and may be an additional point of research in the future. However, these preliminary findings suggest that the actual communication of fleeting relationships is increasingly taking place online and in digital contexts, specifically on the YouTube application, and through 'Story Time' videos. Accordingly, the main takeaway in these initial findings/response to RQ1 is that individuals are increasingly taking to digital platforms to communicate the experience of modern, fleeting romance. Through an analysis of this nature, we can conclude that online video sharing platforms represent a site of self-actualization and community for vloggers in which, through 
the act of digital storytelling, individuals form networks of identity and presence (Santos et al., 2007). Furthermore, we can interpret the use of the digital storytelling format to engage online users through specific acts of digital interaction, shared purpose and shared culture (Rotman \& Preece, 2010). Thus, by sharing the experience of being ghosted on their channels, the vloggers are effectively contributing to a shared online culture with regard to the experience of fleeting romance.

Moreover, as scholar Clement Chau (2010) describes, websites such as YouTube "combine media production and distribution with social networking features, making them an ideal place to create, connect, collaborate, and circulate novel and personally meaningful media" (p. 65). As a result, new media platforms such as YouTube offer a "participatory culture in which to develop, interact, and learn" (p. 65). This description of YouTube as a participatory platform has proved useful to the current study. Considering the common use of YouTube to communicate the act of fleeting intimacies, we can conclude that digital sharing/participatory platforms are increasingly being favoured as the popular modes of communicating, sharing, learning and relating to one another in a modern setting.

Regarding RQ2, the author was interested in the question: "Where is the communication of fleeting online relationships taking place?". This question was posed in order to understand how space and location may influence the communication of fleeting relationships, and furthermore, how these experiences were being framed within particular spaces.

Thus, as previously established, the communication of fleeting online relationships is taking place on the digital networking platform, YouTube. However, the specific locations in which the vloggers recounted their ghosting experiences proved of additional interest for the author. To this degree, the author noticed many commonalities with regard to the spaces in which these 'Story Time' vlogs were being filmed, spaces that are typically considered private and intimate spaces in an 
individual's home and/or day-to-day life. Of all the videos sampled: 6 of the 11 videos were recorded in the vloggers' bedrooms, 3 were recorded in the vloggers' living room, and 1 was recorded in the vlogger's car. In all these spaces, the vloggers were filmed alone, speaking frankly and directly to the camera about their experience of being ghosted. Accordingly, these finding suggest that the communication of fleeting romance is most enacted via private spaces, whereby the vlogger is filmed always alone, and in personal settings. Whether these spaces were intentionally chosen for their private features remains to be seen. However, given the intimate nature surrounding the nature of these conversations, it may be safe to assume that these locations are specifically chosen for the ability to speak more candidly and openly about their personal experiences within these spaces.

Based on these preliminary spatial findings, we can conclude that the communication of fleeting relationships is taking place online and through the YouTube app, and specifically in private spaces such as the vlogger's bedroom, living room or car. In turn, these findings suggest that the communication of personal experiences related to acts of romantic intimacy are most commonly recorded in private spaces. To this degree, we can assume that the communication of romantic intimacy remains a personal subject to be disclosed in comfortable and private settings, albeit and somewhat paradoxically, shared in open digital spaces, to be watched and consumed by a public audience on YouTube.

Moving forward, the author was interested in uncovering both the messages communicated and emotions felt by each vlogger within the shared digital space. The author felt that an analysis of the messages communicated, and emotions felt within these spaces was necessary in order to fully grasp the extent to which fleeting relationships are felt and experienced in a modern setting, which lead the author to the sub question: "How are fleeting relationships being conceptualized in these spaces?". By spaces, the author means to describe not only the YouTube platform in which the 'Story Times' are 
being disseminated, but equally the virtual locations from which the vlogs themselves were being filmed.

To this degree, some notable findings were discovered with regard to the communication of fleeting intimacy in a modern, digital setting. In particular, the author was struck with the notion that there were two primary modes of conceptualizing being ghosted by mobile dating app partners within these spaces. Specifically, group 1 (negative affect group, 7/11 vloggers) had spoken of the frustration and demoralizing element of being active on mobile dating apps, and subsequently, of having to go through the process of being ghosted by their online romantic connections. In this demoralized group context, the vloggers often spoke from a space of emotional frustration, anger and grief, as if they were mourning the loss of something incredibly personal and deeply painful. Despite the minimal interactions these individuals experienced with their romantic partners (either all via digital communications or for a total of 2 in-person dates), these vloggers often spoke of being ghosted from a place of confusion, frustration and hurt. The group spoke directly on their negative feelings in the context of online dating, with some messages ranging from "I am never dating anyone ever again", to others communicating feeling "sad" and "fucked up" over the lack of communication, truth, and emotionally transparency from their romantic partners.

Speaking candidly about their emotions regarding the ghosting experience, one vlogger explains, how “[Being ghosted] really sucked. I think that's something that's really shady to do... I like people that are just straight forward and blunt, and if you're not into me or not into it, just say so, and feel free to do whatever you want." These particular comments reflect similar messages expressed by other hurt/defeated vloggers in group 1, in which the values of honesty are emphasized and sought out in their future partners. Often, the messages communicated within these videos express a willingness to continue to participate in the dating scene, albeit, with little to no patience for future temporary or 
transient ghosting experiences. Furthermore, another 'hurt' vlogger spoke of her feelings of confusion with regard to being ghosted, stating that: "there was no communication of what went wrong", and if she had done something wrong, she "would have wanted to know". In this video, specific feelings of perplexity are described, with the vlogger further discussing of the dilemma of online dating, and how the 'ghostee' is often left to speculate what went wrong within the relationship as a result of the lack of direct communication from their partner.

These feelings of conclusion and lack of closure reflect additional sentiments made by a third vlogger in group 1 in which the process of being ghosted is described as a "frustrating experience". As the vlogger explains, "I hate having my time wasted... I prefer transparency to excuses.... I would rather know straight up what happened, instead of wondering what I did to make them stop responding to me". Similar to vlogger 2, these feelings of confusion point to the more insidious nature of ghosting, in which the individual is often left feeling hurt, confused and frustrated after being ghosted. Accordingly, the emotions described by this group of vloggers suggests that individuals communicate feelings of pain, anguish, and loss with regard to fleeting romance, and specifically, through digital spaces.

Conversely, a smaller number of vloggers (3/11), had spoken of their experience of being ghosted as a normalized experience, one in which they had learned to simply brush off and come to accept over time. These vloggers in group 2 often operated from a place of self-acceptance and emotionally maturity, subsequently describing the process of being ghosted as something to be expected within today's current dating culture. In these vlogs, sentiments such as "I'm used to it", and "it's fine" were communicated, with one vlogger describing the process of being ghosted as a universal experience and furthermore, something to "laugh at". In these videos, most vloggers had spoken about ghosting as a potential challenge to face within the digital age, but equally, did not regard being 
ghosted as something to fret about. The vloggers often expressed that being ghosted was merely a reflection of the other individual, and of normalized processes of conflict avoidance in today's digital age, and thus, did not have to do with any specific problems with the 'ghostee' themselves. These feeling of self-actualization and self-love came up often throughout the study and point to the more optimistic and positive outlook regarding the experience of being ghosted.

Throughout each video, both group 1 and 2 had come to accept their experiences as a reflection of the shortcomings of their partners, often urging their subscribers not to internalize feelings of selfdoubt after being ghosted. As one vlogger saliently describes, "Don't take being ghosted personally. Clearly, the person ghosting you is not mature enough or emotionally intelligent enough to have an open conversation with you, and you deserve better than that." This sentiment was reiterated often throughout the sampled videos, whereby the vloggers express that being ghosted is not something to "be ashamed of", and furthermore, something that most "individuals will experience today". Thus, considering the apparent normalization of ghosting in today's digital dating age, messages of empowerment were disseminated by these vloggers. Even the most 'hurt' vloggers emphasized the idea that being ghosted is not a reflection of anything that the 'ghostee' has done, and simply a reflection of minimal communication skills by their romantic partners.

Ultimately, these findings suggest that the communication of fleeting romance is taking place online and on the YouTube platform. Furthermore, with regard to the actual spatial features of the vlogs, it was noted that all videos were recorded in personal, private and intimate spaces. Based on these findings, we can conclude that private spaces are often favoured by lifestyle vloggers when recording their vlogs. In addition, we can assume that these spaces are often chosen for their ability to speak more frankly and openly about personal and intimate experiences. The private settings may thus be chosen for their ability to speak on intense and personal emotions and experiences. To this degree, 
and in terms of the emotions and messages communicated within these spaces, two primary sentiments were expressed. On the one hand, a larger amount of vloggers in the study (group 1) had communicated feelings of pain, loss, confusion and frustration with regard to the experience of being ghosted. Within this group, feelings of demoralization and frustration in the context of modern dating were aptly communicated, with individuals often speaking on the frustrating and confusing elements surrounding the act of ghosting, and the subsequent discouraging experience of fleeting romances. On the other hand, a smaller number of vloggers (group 2) had spoken of the normalization of ghosting experiences in today's digital dating age, often brushing these situations off with ease, humor and self-acceptance. This group seemed to conceptualize ghosting as an expected experience in today's society, and not to expend any additional emotions or time on. In each case, both groups emphasized the notion that being ghosted does not reflect on the individual being ghosted, but rather, on the ghoster themselves and thus, not something to be ashamed of or internalize on their own behalf.

Regarding these findings, we can conclude that ghosting experiences are increasingly normalized in today's digital societies, and furthermore, that experiences of fleeting romance are conceptualized as both frustrating and hurtful situations, but equally common experiences not to take personally in today's digital dating age. To this degree, these findings confirm recent studies in which "online dating is one of the most common ways for adults of all ages to find a romantic partner" (Davis \& Fingerman, 2016). The ways in which this common and increasingly popular mode of meeting a romantic partner, and the ways in which these experiences are increasingly communicated as fleeting, has thus been explained. Accordingly, these preliminary findings suggest that as a dominant mode of meeting and engaging in intimate relationships, fleeting romances are to be commonly expected, and can range from feelings of confusion and frustration to 'paying it no mind'. Moreover, we can understand the engagement in modern digital dating practices as an ever-changing and ephemeral 
phenomenon, in which the emotions felt range from positive to negative affects, and are increasingly framed as fleeting in nature.

Moving on to RQ3, the researcher was interested in the question: "Is there a gendered discrepancy between who is ending online relationships, causing them to become fleeting in nature?". This question was posed in order to consider and, further, observe any gendered patterns present in the video content analysis, as well as to make note of who is more commonly doing the ghosting and who is more commonly the ghosted in a digital context. Although the author is aware that an analysis of this nature does not account for the complete gendered nuances of ghosting practices in today's societies, this question was posed directly in relation to the gendered patterns observed with regard to the sampled 'Story Time' videos. To this degree, the gendered analysis proved useful for the author, as it allowed them to more thoroughly conceptualize the ways in which the sampled individuals respond to being ghosted within a specific dating dynamic. In addition, it allowed the author to come to some salient findings with regard to the ways individuals deal with the act of ghosting, and whether this does or does not differ within a queer or heterosexual partnership. Accordingly, the gendered findings were as follows:

In reference to the 11 Story Time videos sampled by the author, 10 of the vloggers were women speaking on their experience of being ghosted by a man on a dating app, with a single video being published by a male vlogger discussing his experience of being ghosted by a woman. Furthermore, of all the videos sampled, each vlogger had recounted their experiences of being ghosted within a heterosexual context, apart from a single female vlogger who identified as a lesbian and spoke of her experiences of being ghosted by another female dating app user. Based on this initial data, we can conclude that most individuals doing the ghosting within this context were heterosexual men, with 
heterosexual women specifically speaking directly on these experiences. Whether this points to a larger phenomenon of men engaging in ghosting acts within heterosexual dating dynamics remains inconclusive. However, this initial data presents an overwhelming discrepancy in terms of the experience of being ghosted when engaging in particular gendered partnerships, with heterosexual men being the primary perpetrators of ghosting.

Of the various videos sampled, one vlogger in particular spoke saliently of the gendered experience of using mobile dating apps and subsequent experiences of ghosting, explaining that next time she saves a man's number in her phone, she will save their contact's name as "Pinocchio", because they are all "liars that will end up disappointing and ghosting you." The vlogger then proceeds to go on a self-professed rant about the millennial generation and online dating. In particular, the vlogger addressed her audience to describe the ways in which online dating can be a temporary, demoralizing, and frustrating experience, while equally communicating that as a woman, you must protect yourself and recognize your own worth in order to not let "these ghosting experiences and men get to you". Accordingly, the sentiments expressed by this user may point to a more universal experience of frustration within heterosexual dating dynamics, as well as the common experience of being deceived and ghosted by men. Considering the amount of female vloggers who had communicated their experiences of being ghosted by men, additional studies on the gendered statistics of ghosting could prove of additional interest for the author moving forward.

These findings were quite surprising for the author, who imagined that in today's technologically mediated society, no one gender is immune to the practices of ghosting. Albeit, the author recognizes that this research does not present any conclusive findings on the actual statistics of gendered ghosting practices present in today's technologically mediated societies. However, these findings may speak more saliently to the idea that women feel more comfortable speaking on these 
experiences and/or relying on the vlogging medium to communicate the experience of being ghosted by their dating app partners. Similar to previous studies on the gender and subject matter of vloggers, this analysis speaks to the idea that female vloggers are more likely to vlog about personal matters than male vloggers, with more women creating vlogs that interact directly with the YouTube community (Molyneaux et al., 2008). The reasons for this may vary but may speak to a larger cultural context of men being discouraged from expressing or sharing their emotions with others, and subsequently, a hesitancy to reveal details about their intimate relationships online. As a result, women vloggers may feel more comfortable taking to online mediums to share their experiences both to, and through, the YouTube community.

In a similar vein, the author was interested in understanding what exactly the gendered communication of ghosting might reveal with regard to modern communication practices. Accordingly, the sub question was raised: "What can a gendered analysis of fleeting relationships tell us about the experience of modern digital communications?". When considering the above findings, we can note that a gendered analysis of fleeting online relationships presents us with the reality that it is predominantly women speaking on these experiences within a digital context. Specifically, we can conclude that women are the most common demographic speaking to the experience of being ghosted while using the YouTube application. Consequently, these findings may point to an increasing trend of women taking to online vlogging mediums to share and recount their personal life stories and experiences with an online community. Such modes of sharing and relating to other YouTube users (whether exclusively with a female or male audience remains to be seen), may thus point to a new and favoured form of connection and community in a modern digital setting for female users. 


\section{Conclusion}

\section{- Main Takeaways}

Throughout the study, several conclusions were drawn. In reference to RQ1, we can conclude that the experience of fleeting romance is being recounted and responded to through specific oral communication measures. In particular, they are recounted via the oral 'Story Time' vlog genre on YouTube. This form of sharing and communicating personal experiences with other online users may thus point to a new form of community experienced in today's digital age. Making up for the lack of communication by their dating app partner, the act of YouTube lifestyle vlogging may represent a new way to conceptualize and communicate experiences of online dating, and further serve as an alternative medium of connection for individuals battling loneliness and reckoning with their experiences of fleeting romance. These findings are of significant interest for the author as they may point to a new and increasingly popular mode of engaging with others online and the dissemination of discourses surrounding fleeting online romance and the use of mobile dating apps to date. Having access to these videos may subsequently ease feelings of loneliness for those that have been ghosted and point to a stronger form of digital community and the process of sharing and relatability of said experiences in a modern digital environment.

With regard to $\mathbf{R Q 2}$, we can conclude that the communication of fleeting relationships is commonly recorded and enacted in private spaces, pointing to the more private and personal communication of intimacy in a digital setting. In these particular settings, the communication of fleeting intimacy was expressed in two ways. On the one hand, the experience of being ghosted was described as a demoralizing, hurtful, confusing and frustrating experience. On the other, ghosting was described as a common, normal and inconsequential experience of today's digitally mediated dating 
age. These findings are significant as they point to the complexities of emotions and affect felt with regard to the experience of fleeting romance and being ghosted, in particular. Understanding the various emotions felt within these experiences of fleeting romance may explain how online dating informs and impacts individuals in a contemporary setting and further point to the more negative and insidious impacts of the use of mobile dating apps. They may also point to the commonality of these experiences today and serve as a useful point of reference for individuals navigating the current online dating scene.

Finally, and regarding RQ3, we can conclude that based on the sampled videos, it is primarily heterosexual men that are ghosting their romantic partners, thus causing them to become fleeting in nature. Whether this points to a larger heterosexual phenomenon of ghosting remains to be seen. However, within this sample, we can effectively conclude that it is primarily women vloggers who are recounting their experiences of being ghosted online, and thus, that it is men that are the primary arbiters of fleeting relationships within the study. These findings are significant as they may point to an increasing gendered phenomenon of women taking to online mediums such as YouTube to both express and share their personal experiences through the lifestyle vlog. Investigating the way experiences of fleeting romance are communicated by specific demographic and gendered groups on YouTube may reveal significant insights into who is most commonly communicating acts of ghosting and personal experiences related to online dating in a contemporary, digital setting.

\section{- Limitations}

Certain limitations presented themselves to the author throughout the study. As the author began their YouTube video search, they were confronted with the reality that there were not a significant number of videos relating directly to the experience of being ghosted by partners vloggers 
they had met on dating apps. Very few YouTube 'Story Time' vlogs dealt directly with the experience of being dating on the particular apps (Tinder, Bumble, Hinge). Although there were a select few videos from which the researcher based their study on, the initial search found that there were more videos dealing with general stories of being ghosted by partners, most of whom had met organically or through other means or other dating apps other than the ones listed above. In addition, most videos on YouTube dealing with the topic of being ghosted were not in the form of story time videos, but rather took the form of various self-help videos. Furthermore, the author found that individuals discussing the particular experience of fleeting intimacies were taking to additional social media platforms, such as Twitter and TikTok. Some of these accounts offered more detailed explanations of fleeting intimacies and more in-depth communication of acts of ghosting than the ones presented on the YouTube platform through story time videos. These additional findings and subsequent limitations of the YouTube platform point to another possible area of interest for the author, being: what digital medium are users using to communicate the act of fleeting intimacy, and what messages are being communicated on these platforms? Nevertheless, the videos analyzed proved a useful initial point of research for the study and were able to respond to the three research questions on initially a more broad and introductory level. Furthermore, expanding the research questions by two allowed the author to more thoroughly investigate the communication of fleeting romances through a more nuanced lens, with the addition of gendered and spatial considerations.

\section{- Additional Studies}

Moving forward, the author recognizes additional opportunities for further research within the context and study of fleeting relationships. In particular, the gendered and sexual dynamics of fleeting relationships could be further studied in order to understand amongst what gendered and potentially, which sexual lines experiences of ghosting are more commonly experienced, if at all. To this degree, a 
more focused demographic could be studied in order to observe not only the gendered and sexual impacts of ghosting, but equally, the age groups to determine if the experience of fleeting romance is experienced and/or communicated by a particular age demographic. Moreover, an analysis of the YouTube Story Time genre and direct interviews with lifestyle vloggers could be studied in order to further investigate and understand why individuals choose to take to online and digital mediums to communicate their personal experiences and intimate exchanges. An analysis of this nature could reveal a significant amount of information regarding the communication of fleeting intimacy in a modern setting, as well as the various new forms of communication and community in a modern digital environment. In particular, understanding the ways individuals choose to make sense of particular digital phenomena, and specifically with regard to the experience of modern dating, represents a high point of interest for the author, and may reveal additional data on the ways individuals relate to one another in a modern and digital setting. 


\section{References}

Anderson, K. E. (2010). Storytelling. SAGE. https://doi.org/10.7282/T35T3HSK

Apostolou, M., Matogian, I., Koskeridou, G., Shialos, M., \& Georgiadou, P. (2019). The Price of Singlehood: Assessing the Impact of Involuntary Singlehood on Emotions and Life Satisfaction. Evolutionary Psychological Science, 5(4), 416-425. https://doi.org/10.1007/s40806-019-00199-9

Ashton, D., \& Patel, K. (2018). Vlogging Careers: Everyday Expertise, Collaboration and Authenticity. In S. Taylor \& S. Luckman (Eds.), The New Normal of Working Lives (pp. 147169). Springer International Publishing. https://doi.org/10.1007/978-3-319-66038-7_8

Augustine, S. (2008). "Oral Histories and Oral Traditions," in Aboriginal Oral Traditions: Theory, Practice, Ethics, ed. Renée Hulan and Renate Eigenbrod (pp. 2-3). Fernwood Pub.

Basso, K. H. (1996). Wisdom Sits in Places Landscape and Language among the Western Apache. University of New Mexico Press. http://public.eblib.com/choice/PublicFullRecord.aspx?p=6299655

Berryman, R. (2020). "Vloggers" in The International Encyclopedia of Gender, Media, and Communication (1st ed.). Wiley. https://doi.org/10.1002/9781119429128

Brown, E. (2017). It's Storytime. The Outline. Retrieved June 27, 2021, from https://theoutline.com/post/1189/storytime-youtube-where-people-confess-everything-andnothing

Bruchac, M. (2014). Indigenous Knowledge and Traditional Knowledge. Encyclopedia of Global Archaeology, 3814-3824.

Burgess, J., \& Green, J. (2018). YouTube: Online video and participatory culture (Second edition). Polity Press.

Castro, Á., Barrada, J. R., Ramos-Villagrasa, P. J., \& Fernández-del-Río, E. (2020). Profiling Dating Apps Users: Sociodemographic and Personality Characteristics. International Journal of Environmental Research and Public Health, 17(10), 3653. https://doi.org/10.3390/ijerph17103653 
Chau, C. (2010). YouTube as a participatory culture. New Directions for Youth Development, 2010(128), 65-74. https://doi.org/10.1002/yd.376

Chen, G. M. (2012). The Impact of New Media on Intercultural Communication in Global Context. 11.

Cigna-2020-loneliness-factsheet.pdf. (2018). Retrieved June 22, 2021, from https://www.cigna.com/static/www-cigna-com/docs/about-us/newsroom/studies-andreports/combatting-loneliness/cigna-2020-loneliness-factsheet.pdf

Collins, P., \& Gallinat, A. (2013). The Ethnographic Self as Resource: Writing Memory and Experience into Ethnography. Berghahn Books.

Cruikshank, J. (2014). Do Glaciers Listen?: Local Knowledge, Colonial Encounters, and Social Imagination. UBC Press.

http://public.ebookcentral.proquest.com/choice/publicfullrecord.aspx?p=3412087

Davis, E. M., \& Fingerman, K. L. (2016). Digital Dating: Online Profile Content of Older and Younger Adults. The Journals of Gerontology Series B: Psychological Sciences and Social Sciences, 71(6), 959-967. https://doi.org/10.1093/geronb/gbv042

Delevi, R., \& Weisskirch, R. S. (2013). Personality factors as predictors of sexting. Computers in Human Behavior, 29(6), 2589-2594. https://doi.org/10.1016/j.chb.2013.06.003

Dreon, O., Kerper, R. M., \& Landis, J. (2011). Digital Storytelling: A Tool for Teaching and Learning in the YouTube Generation. Middle School Journal, 42(5), 4-10. https://doi.org/10.1080/00940771.2011.11461777

Eigenbrod, R., \& Hulan, R. (Eds.). (2008). Aboriginal oral traditions: Theory, practice, Ethics. Fernwood Pub.

Erevik, E. K., Kristensen, J. H., Torsheim, T., Vedaa, Ø., \& Pallesen, S. (2020). Tinder Use and Romantic Relationship Formations: A Large-Scale Longitudinal Study. Frontiers in Psychology, 11, 1757. https://doi.org/10.3389/fpsyg.2020.01757 
Everingham, R. (2019, November 24). Ghosting - The Technological Embodiment of Avoidance. Mannaz Therapy Space. https://www.mannaztherapy.com/ghosting-the-technologicalembodiment-of-avoidance/

Finkel, E. J., Eastwick, P. W., Karney, B. R., Reis, H. T., \& Sprecher, S. (2012). Online Dating: A Critical Analysis From the Perspective of Psychological Science. Psychological Science in the Public Interest, 13(1), 3-66. https://doi.org/10.1177/1529100612436522

Frampton, J. R., \& Fox, J. (2018). Social Media’s Role in Romantic Partners' Retroactive Jealousy: Social Comparison, Uncertainty, and Information Seeking. Social Media + Society, 4(3), 2056305118800317. https://doi.org/10.1177/2056305118800317

Freedman, G., Powell, D. N., Le, B., \& Williams, K. D. (2019). Ghosting and destiny: Implicit theories of relationships predict beliefs about ghosting. Journal of Social and Personal Relationships, 36(3), 905-924. https://doi.org/10.1177/0265407517748791

Gatter, K., \& Hodkinson, K. (2016). On the differences between Tinder $^{\mathrm{TM}}$ versus online dating agencies: Questioning a myth. An exploratory study. Cogent Psychology, 3(1), 1162414. https://doi.org/10.1080/23311908.2016.1162414

Giles-Vernick, T. (2006). Oral Histories: Oral Histories as Methods and Sources. In E. Perecman \& S. Curran, A Handbook for Social Science Field Research: Essays \& Bibliographic Sources on Research Design and Methods (pp. 85-102). SAGE Publications, Inc. https://doi.org/10.4135/9781412983211.n4

Hanson, E. (2009). Oral Traditions. Oral Traditions. https://indigenousfoundations.arts.ubc.ca/oral_traditions/

Hardey, M. (2004). Mediated Relationships. Information, Communication \& Society, 7(2), 207 - 222. https://doi.org/10.1080/1369118042000232657

Hogan, B., li, nai, \& Dutton, W. H. (2011). A Global Shift in the Social Relationships of Networked Individuals: Meeting and Dating Online Comes of Age. SSRN Electronic Journal. https://doi.org/10.2139/ssrn.1763884 
Koessler, R. B., Kohut, T., \& Campbell, L. (2019). When Your Boo Becomes a Ghost: The Association Between Breakup Strategy and Breakup Role in Experiences of Relationship Dissolution. Collabra: Psychology, 5(1), 29. https://doi.org/10.1525/collabra.230

Kwok, I., \& Wescott, A. B. (2020). Cyberintimacy: A Scoping Review of Technology-Mediated Romance in the Digital Age. Cyberpsychology, Behavior, and Social Networking, 23(10), 657-666. https://doi.org/10.1089/cyber.2019.0764

Lang, M., Laing, C., Moules, N., \& Estefan, A. (2019). Words, Camera, Music, Action: A Methodology of Digital Storytelling in a Health Care Setting. International Journal of Qualitative Methods, 18, 160940691986324. https://doi.org/10.1177/1609406919863241

Langellier, K. M. (1989). Personal narratives: Perspectives on theory and research. Text and Performance Quarterly, 9(4), 243-276. https://doi.org/10.1080/10462938909365938

LeFebvre, L. (2017). Phantom Lovers: Ghosting as a Relationship Dissolution Strategy in the Technological Age (pp. 219-236).

LeFebvre, L. E., Allen, M., Rasner, R. D., Garstad, S., Wilms, A., \& Parrish, C. (2019). Ghosting in Emerging Adults' Romantic Relationships: The Digital Dissolution Disappearance Strategy. Imagination, Cognition and Personality, 39(2), 125-150. https://doi.org/10.1177/0276236618820519

Lomanowska, A. M., \& Guitton, M. J. (2016). Online intimacy and well-being in the digital age. Internet Interventions, 4, 138-144. https://doi.org/10.1016/j.invent.2016.06.005

Loneliness in America: How the Pandemic Has Deepened an Epidemic of Loneliness and What We Can Do About It. (2021). Making Caring Common. Retrieved July 6, 2021, from https://mcc.gse.harvard.edu/reports/loneliness-in-america

McAdams, D. P. (1999). Personal narratives and the life story. In Handbook of personality: Theory and research, 2nd ed (pp. 478-500). Guilford Press.

Molyneaux, H., O’Donnell, S., Gibson, K., \& Singer, J. (2008). Exploring the gender divide on YouTube: An analysis of the creation and reception of vlogs. American Communication Journal, 10. 
Morrill, C., Snow, D. A., \& White, C. H. (Eds.). (2005). Together alone: Personal relationships in public places. University of California Press.

Navarro, R., Larrañaga, E., Yubero, S., \& Víllora, B. (2020). Psychological Correlates of Ghosting and Breadcrumbing Experiences: A Preliminary Study among Adults. International Journal of Environmental Research and Public Health, 17(3), 1116.

https://doi.org/10.3390/ijerph17031116

Neyt, B., Baert, S., \& Vandenbulcke, S. (2019). Never mind I'll find someone like me - Assortative mating preferences on Tinder. Personality and Individual Differences, 155. https://doi.org/10.1016/j.paid.2019.109739

Online Dating - Worldwide| Statista Market Forecast. (2019). Statista. https://www.statista.com/outlook/dmo/eservices/dating-services/online-dating/worldwide

Öze, N. (2017). Communication And Devices: Face To Face Communication Versus Communication With Mobile Technologies. https://doi.org/10.5281/ZENODO.1130519

Pittman, M. (2015). Creating, Consuming, and Connecting: Examining the Relationship Between Social Media Engagement and Loneliness. New Media \& Society, 4.

Punyanunt-Carter, N., \& Wrench, J. S. (Eds.). (2017). The impact of social media in modern romantic relationships. Lexington Books.

Quiroz, P. A. (2013). From Finding the Perfect Love Online to Satellite Dating and 'Loving-the- OneYou're Near': A Look at Grindr, Skout, Plenty of Fish, Meet Moi, Zoosk and Assisted Serendipity. Humanity \& Society.

Ritchie, D. A. (1995). Doing Oral History. Twayne Publishers.

Robin, B. R. (2008). Digital Storytelling: A Powerful Technology Tool for the 21st Century Classroom. Theory Into Practice, 47(3), 220-228. https://doi.org/10.1080/00405840802153916 
Rosenfeld, M. J., Thomas, R. J., \& Hausen, S. (2019). Disintermediating your friends: How online dating in the United States displaces other ways of meeting. Proceedings of the National Academy of Sciences, 116(36), 17753-17758.

Rotman, D., \& Preece, J. (2010). The "WeTube" in YouTube; creating an online community through video sharing. International Journal of Web Based Communities, 6(3), 317. https://doi.org/10.1504/IJWBC.2010.033755

Santos, R, L., Rocha, B, P., Rezende, R, G., and Loureiro, A., A. (2007). Characterizing the YouTube video-sharing community. Technical report.

Sarkisian, N., \& Gerstel, N. (2016). Does singlehood isolate or integrate? Examining the link between marital status and ties to kin, friends, and neighbors. Journal of Social and Personal Relationships, 33(3), 361-384. https://doi.org/10.1177/0265407515597564

Slumkoski, C. (2012). History on the Internet 2.0: The Rise of Social Media. Acadiensis. https://journals.lib.unb.ca/index.php/Acadiensis/article/view/20073

Sutcliffe, A. G., Binder, J. F., \& Dunbar, R. I. M. (2018). Activity in social media and intimacy in social relationships. Computers in Human Behavior, 85, 227-235.

https://doi.org/10.1016/j.chb.2018.03.050

Thompson, P. (2017). The Voice of the Past: Oral History. Oxford University Press.

Wiederhold, B. K. (2015). Twenty Years of Online Dating: Current Psychology and Future Prospects. Cyberpsychology, Behavior, and Social Networking, 18(12), 695-696. https://doi.org/10.1089/cyber.2015.29017.bkw

Wooley, S. (2013). Constantly Connected: The Impact of Social Media and the Advancement in Technology on the Study Abroad Experience. Undefined.

Yeo, T. E. D., \& Fung, T. H. (2018). "Mr Right Now": Temporality of relationship formation on gay mobile dating apps. Mobile Media \& Communication, 6(1), 3-18. https://doi.org/10.1177/2050157917718601 
Yum, Y., \& Hara, K. (2005). Computer-Mediated Relationship Development: A Cross-Cultural Comparison. Journal of Computer-Mediated Communication, 11(1), 133-152. https://doi.org/10.1111/j.1083-6101.2006.tb00307.x 